\title{
Auger-TA energy spectrum working group report
}

\author{
Tareq AbuZayyad ${ }^{1}$, Olivier Deligny ${ }^{2}$, Daisuke Ikeda $^{3}$, Dmitri Ivanov ${ }^{1}$, Isabelle Lhenry-Yvon ${ }^{2}$, Ioana Mariş ${ }^{4}$, Daniela \\ Mockler $^{8}$, Toshiyuki Nonaka ${ }^{3}$, Markus Roth ${ }^{5}$, Francesco Salamida ${ }^{6,7}$, Gordon Thomson ${ }^{1}$, Yoshiki Tsunesada ${ }^{9}$, Inés \\ Valiño ${ }^{10,11}$, and Valerio Verzi ${ }^{12}$, for the Pierre Auger ${ }^{13}$ and Telescope Array ${ }^{14}$ Collaborations \\ ${ }^{1}$ University of Utah, High Energy Astrophysics Institute, Salt Lake City, Utah, USA \\ ${ }^{2}$ Institut de Physique Nucléaire, CNRS-IN2P3, Univ. Paris-Sud, Université Paris-Saclay, 91406 Orsay Cedex, France \\ ${ }^{3}$ Institute for Cosmic Ray Research, University of Tokyo, Kashiwa, Chiba, Japan \\ ${ }^{4}$ Université Libre de Bruxelles, Belgium \\ ${ }^{5}$ Karlsruhe Institute of Technology, Institut für Kernphysik (IKP), Karlsruhe, Germany \\ ${ }^{6}$ Dipartimento di Scienze Fisiche e Chimiche, Università dell'Aquila, L'Aquila, Italy \\ ${ }^{7}$ INFN Laboratori Nazionali del Gran Sasso, Assergi (L'Aquila), Italy \\ ${ }^{8}$ Karlsruhe Institute of Technology, Institut für Experimentelle Kernphysik (IEKP), Karlsruhe, Germany \\ ${ }^{9}$ Graduate School of Science, Osaka City University, Osaka, Japan \\ ${ }^{10}$ INFN Laboratori Nazionali del Gran Sasso, Assergi (L'Aquila), Italy \\ ${ }^{11}$ Gran Sasso Science Institute, L'Aquila, Italy \\ ${ }^{12}$ Sezione INFN di Roma "Tor Vergata", Roma, Italy \\ ${ }^{13}$ Observatorio Pierre Auger, Av. San Martín Norte 304, 5613 Malargüe, Argentina \\ Full author list: http://www.auger.org/archive/authors_2018_10.html \\ ${ }^{14}$ Telescope Array Project, 201 James Fletcher Bldg, 115 S. 1400 East, Salt Lake City, UT 84112-0830, USA \\ Full author list: http://www.telescopearray.org/index.php/research/collaborators
}

\begin{abstract}
The energy spectrum of ultra-high energy cosmic rays is the most emblematic observable for describing these particles. Beyond a few tens of EeV, the Pierre Auger Observatory and the Telescope Array, currently being exploited, provide the largest exposures ever accumulated in the Southern and Northern hemispheres to measure independently a suppression of the intensity, in a complementary way in terms of the coverage of the sky. However, the comparison of the spectra shows differences that are not reducible to an overall uncertainty on the calibration of the energy scale used to reconstruct the extensive air showers. In line with the previous editions of the UHECR workshops, a working group common to both experiments examined these differences by focusing this time on quantification of these differences in the region of the sky commonly observed, where the spectra should be in agreement within uncertainties when directional-exposure effects are taken into account. These differences are compared with the systematic uncertainties of each experiment. We have also revisited the methods of determining cosmic-ray energies and deriving the energy spectrum. We describe the surface detector (SD) spectrum obtained adopting an energy calibration based on the constant intensity cut method (CIC), a Monte Carlo-based attenuation correction, and an energy-dependent CIC attenuation correction.
\end{abstract}

\section{Introduction}

A good measurement of the cosmic-ray energy spectrum at the highest energies is essential for our understanding of the ultrahigh energy cosmic rays (UHECR), their sources, and their propagation mechanisms. In an effort to achieve this goal, two experiments of unprecedented size and precision, have been operating over the past decade: the Pierre Auger Observatory (Auger) [1], viewing the Southern hemisphere, and the Telescope Array (TA) [24], observing the Northern hemisphere, have been collecting data for more than ten years. Both experiments have delivered their latest spectrum results, and both agree that there is an ankle feature in the spectrum near $\sim 10^{18.5} \mathrm{eV}$ and a suppression near $\sim 10^{19.5} \mathrm{eV}[5,6]$. The precise locations of these features, however, as well as the overall energy scales of the experiments, appeared to differ in significant ways as early as in 2010 when TA and Auger had collected their first two and four years of data, respectively. The TA-Auger differences have raised an important question: are these differences an astrophysical effect, because Auger and TA are viewing different regions of the sky, or can these differences be explained by the systematic uncertainties of the two experiments?

At the first UHECR conference, held in 2010 in Nagoya, Japan, the Telescope Array and Pierre Auger Collaborations formed the Auger-TA energy spectrum working group (WG), with the aim of understanding our methods, the systematic uncertainties, and, ultimately, the differences between our spectra. Other cosmic-ray experiments have also participated in the WG activities. Soon thereafter, at the UHECR 2012 conference at CERN, our methods and results have been compared and cross- 
checked for the first time [7], and at the UHECR 2014, the spectrum WG has conclusively demonstrated that the Auger-TA energy scale difference can be explained by the different fluorescence yield and invisible energy models used by the two experiments.

At the UHECR 2016 and ICRC 2017 [8, 9], we have found that although the TA and Auger results agree in the ankle region of the spectrum, when Auger energies are rescaled by a constant factor of $+5.2 \%$ and TA energies are rescaled by the same amount in the opposite direction, $-5.2 \%$, there is a large difference between the TA and Auger spectra above $10^{19.4} \mathrm{eV}$ that does not disappear after the constant energy rescaling. It was also shown then, by restricting the TA and Auger spectrum measurements to a common declination band, that a declination dependence [10] of the energy spectrum in the Northern hemisphere could be one of the contributing factors to this discrepancy, although it did not account for the entire effect $[8,9]$.

To explain the remaining difference between the Auger and TA energy spectra at the highest energies, we must now examine the energy-dependent systematic uncertainties of the two experiments. In this work, we update the results of the spectrum WG presented at the UHECR 2016 and ICRC 2017, provide a discussion and a summary of the TA and Auger energy-dependent systematic uncertainties and a cross-check of the TA and Auger spectrum results using alternative energy reconstruction methods.

\section{TA and Auger spectra}

The Telescope Array and Pierre Auger Observatory are both hybrid cosmic-ray detectors [1-4] that consist of fluorescence telescopes overviewing an array of surface detectors. Although the fluorescence detectors provide an accurate determination of the cosmic-ray energy, as they see the longitudinal developments of the cosmic-ray air showers, their duty cycle is only about $15 \%$, which makes it advantageous for both Auger and TA to use their surface detectors to measure the energy spectrum at the highest energies. Both Auger and TA calibrate their surface detector (SD) energies to the fluorescence detectors (FD) using cosmic-ray events simultaneously detected by the FD and SD.

Figure 1 shows the TA [10] and Auger [5] energy spectra calculated over their entire fields of view. After adjusting the energy scales of the two experiments, Auger and TA spectra are in good agreement around the ankle region but a large discrepancy above $10^{19.4} \mathrm{eV}$ remains. In the spectrum working group reports $[8,9]$, we have addressed this difference by restricting the measurement of the TA and Auger spectra to the declination band $-15.7^{\circ}<\delta<24.8^{\circ}$ that is covered by both the TA and Auger Observatories and by calculating the spectra using a method that takes into account the declination dependence of the Auger and TA exposure (see [8]). As the left panel of Figure 2 shows, the TA and Auger spectra are in a better agreement after this adjustment, that can be partly explained by a $3.5 \sigma$ declination dependence of the TA spectrum [10]. Although the TA and Auger spectra are in a better agreement after restricting them to the common declination band, a significant difference remains, as the ratio of the two spectra in the right panel of Figure 2 demonstrates. Since now we are looking at the same patch of sky and are using a method that removes the declination dependence of the exposure, we must examine the systematic uncertainties of Auger and TA that can produce energy-dependent effects in our SD spectra. Figure 3 demonstrates that the relative energy-dependent effects, between Auger and TA, are of the order of $20 \%$ per decade: if Auger energies are corrected by a $+10 \%$ per decade nonlinear correction and the TA energies are corrected by $-10 \%$ per decade nonlinear correction then the Auger and TA spectra would agree.

\section{Systematic uncertainties of the TA and Auger energy spectra}

In the previous working group reports $[8,9]$, we have examined the systematic uncertainties of the constant energy scaling factors of Auger and TA. It was then established that for TA, the systematic uncertainty of the overall energy scale is $21 \%$, while for Auger, the energy scale systematic uncertainty is $14 \%$ [11]. We now consider the energy-dependent systematic uncertainties in the analyses of the two experiments. Since the detector types, reconstruction and analysis methods are different for TA and Auger, the approaches for quoting the energy-dependent uncertainties for the two experiments will be somewhat different too. Below, we consider the important categories of the energy-dependent sources of the systematic uncertainties and summarize the results for each experiment.

\subsection{Aerosols}

Since higher energy showers are brighter, the distances from which the fluorescence detectors can see them increase with energy. Because the effects of the atmospheric light attenuation must be taken into account in determining the energy of the shower, an uncertainty in the atmospheric attenuation parameters can lead to an energy-dependent reconstruction bias for the fluorescence detector that can propagate to the calibration of the surface detector energy scale by the fluorescence detector.

Figure 4 shows the systematic check for TA. We generate a Monte Carlo sample of TA hybrid events [12] using the mean vertical aerosol optical depth (VAOD) of 0.04 and then reconstruct these simulated showers using VAOD values of 0.04 and 0.034 , which would be an extreme case scenario for TA. We then plot the natural logarithm of the ratio of the reconstructed and generated energy versus the logarithm of the generated Monte Carlo energy for the values of the VAOD of 0.04 and 0.034 . We find that in the extreme case scenario, the energy-dependent reconstruction bias is only $1.7 \%$ per energy decade for TA.

Figure 5 shows the study of the energy-dependent biases due to the aerosols done by Auger. As can be seen from Figure 5, the energy-dependent bias obtained changing the VAOD by its uncertainty is expected to be of the order of $1 \%$ per decade of energy for Auger. In addition, 

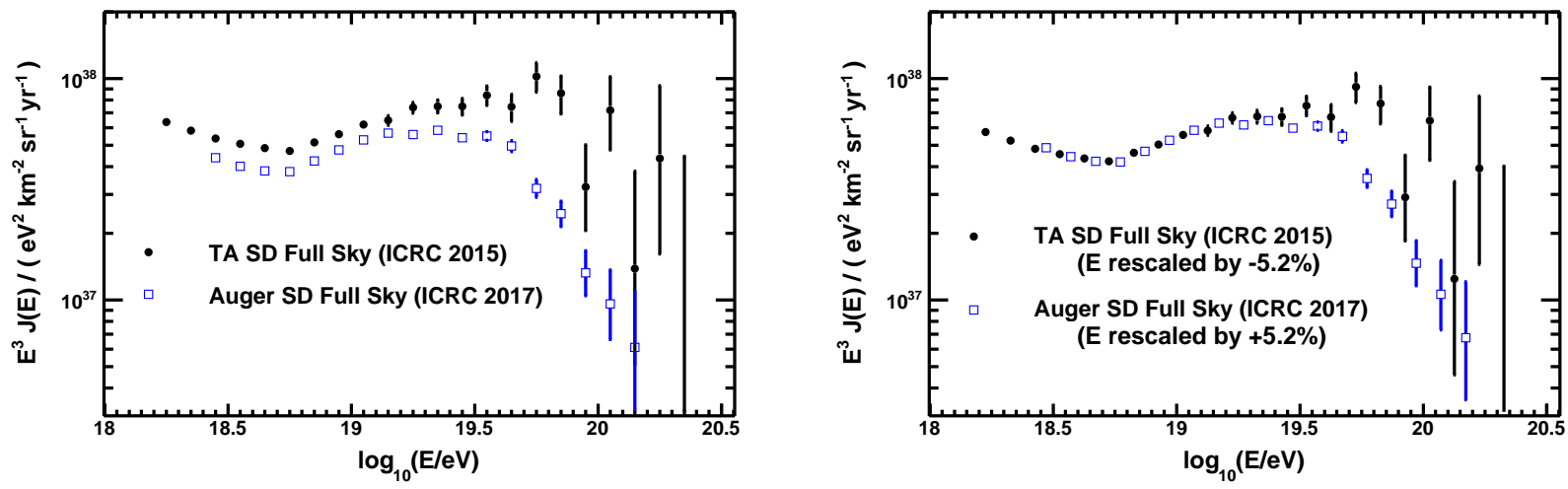

Figure 1. Energy spectra over the entire fields of view for TA [10] and Auger [5]: (left) calculated using the nominal energy scales of TA and Auger, (right) calculated after applying the overall $+5.2 \%$ (Auger) and $-5.2 \%$ (TA) energy scale corrections. Significant difference between the Auger and TA energy spectra remains after rescaling the TA and Auger energies by constant (energy-independent) factors.
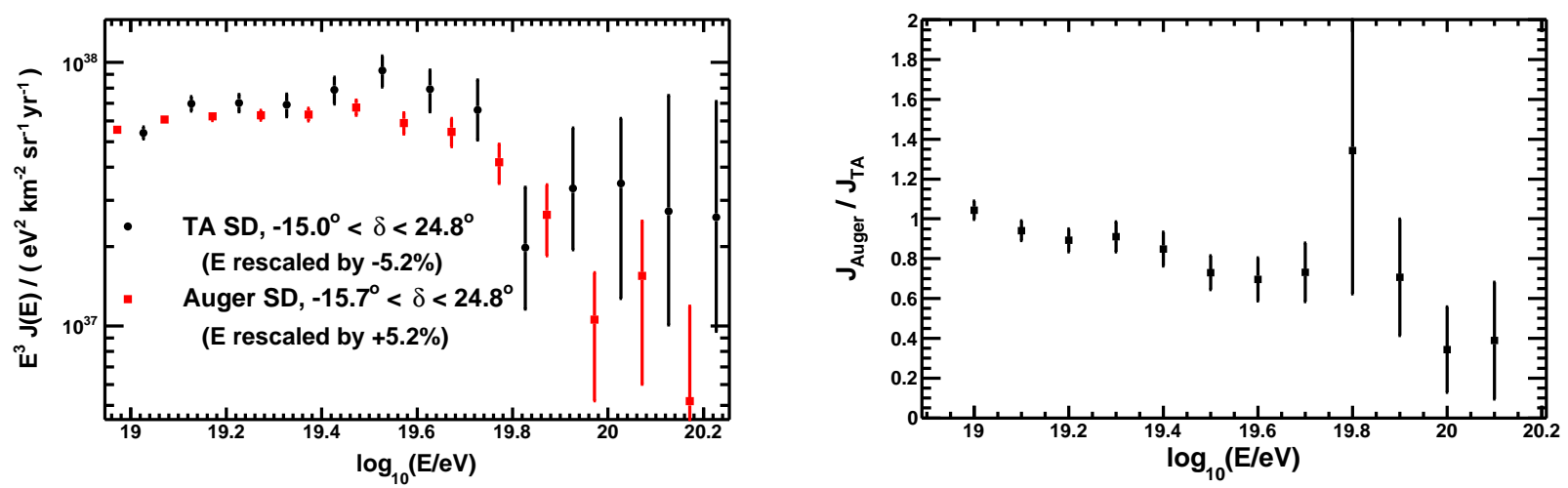

Figure 2. (left) TA and Auger spectra in the common declination band after applying overall $+5.2 \%$ (Auger) and $-5.2 \%$ (TA) energy scale corrections. (right) Significant difference between TA and Auger spectra persists even after rescaling the TA and Auger energies by the constant factors and restricting the comparison to the common declination band.
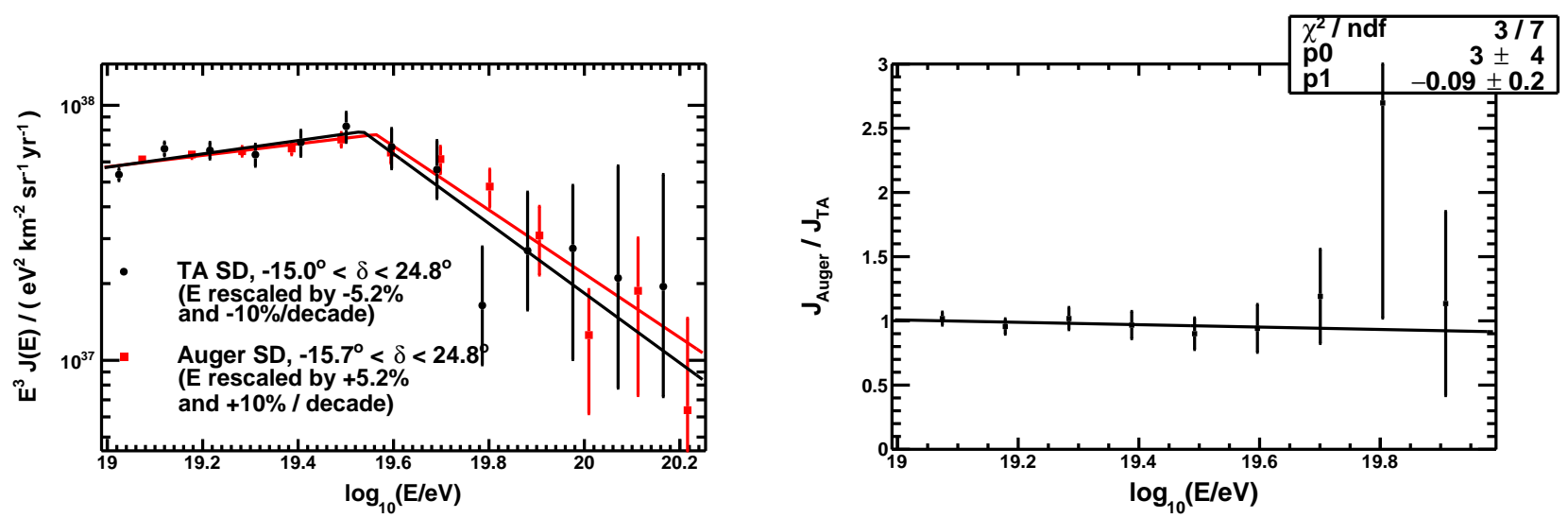

Figure 3. (left) TA and Auger spectra in the common declination band after applying additional energy-dependent energy corrections to TA and Auger. In addition to overall $+5.2 \%$ (Auger) and $-5.2 \%$ (TA) energy scale corrections, TA and Auger energies were corrected by $+10 \%$ / decade (Auger) and $-10 \%$ / decade (TA) starting at $10 \mathrm{EeV}$. (right) After these rescalings of the energies, the TA and Auger spectra are in excellent agreement.

Auger has examined the ratio of the surface detector and the fluorescence detector energies versus the aerosol transmission to the shower maximum. As can be seen from Fig- ure 6, Auger has no apparent energy reconstruction bias with respect to the aerosol transmission [13]. 


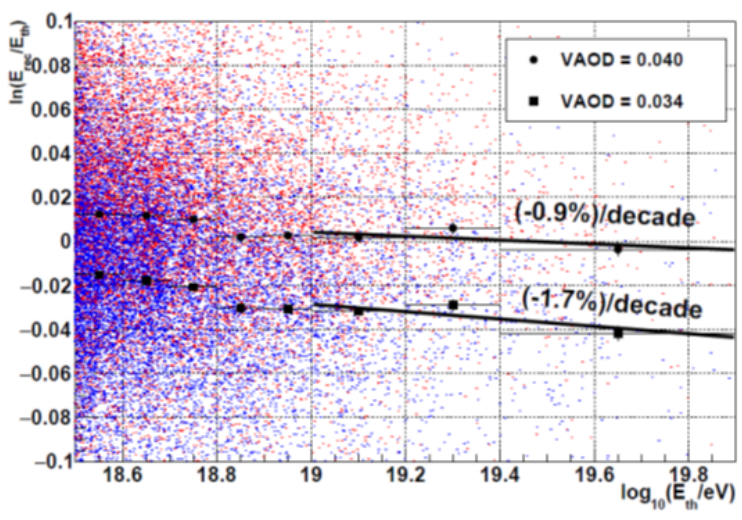

Figure 4. TA energy-dependent reconstruction bias due to the aerosols. Natural logarithm of the ratio of the reconstructed Monte Carlo energy and the thrown (generated) energy, $\ln \left(E_{\mathrm{rec}} / E_{\mathrm{thr}}\right)$ is plotted versus the logarithm of the thrown energy $\log _{10}\left(E_{\mathrm{thr}} / \mathrm{eV}\right)$. Red points correspond to the Monte Carlo reconstruction using VAOD of 0.04 and the blue points correspond to the reconstruction using VAOD of 0.034. The Monte Carlo events were generated with VAOD of 0.04 in both cases. As the linear fits (solid lines) to the mean values of $\ln \left(E_{\text {rec }} / E_{\text {thr }}\right)$ indicate, the bias for the 0.04 VAOD reconstruction (filled circles) is $-0.9 \%$ per decade, and the bias for the VAOD 0.034 reconstruction (filled squares) is $-1.7 \%$ per decade of energy.

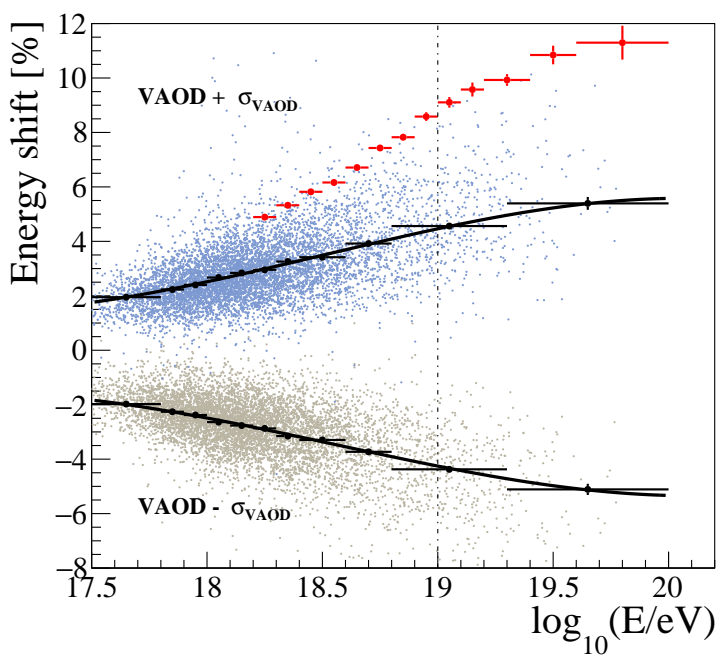

Figure 5. Auger energy-dependent reconstruction bias due to the aerosols. Black curves indicate the expected average energy shift of $1 \%$ per decade above $10 \mathrm{EeV}$ for Auger, if the Auger VAOD is increased (top curve) or decreased (bottom curve) by its uncertainty $\sigma_{\mathrm{VAOD}}$. The dots show the energy shifts for each FD event. Red dots show the expected average energy shift of $2 \%$ per decade above $10 \mathrm{EeV}$ for an extreme case scenario.

All other sources of the TA and Auger FD nonlinearities, such as the fluorescence yield models and the invisible energy corrections, have been already examined in the previous Auger-TA spectrum working group reports [7-9] and do not contribute more than $1 \%$ per decade of energy above $10^{19} \mathrm{eV}$.

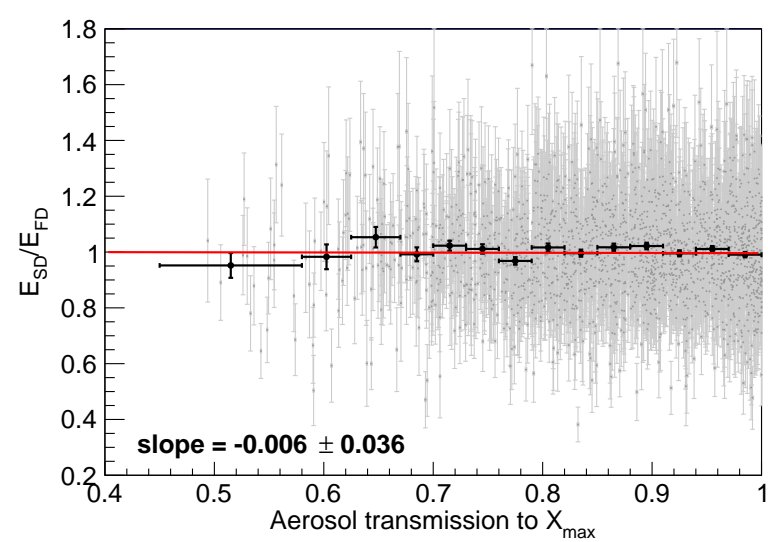

Figure 6. The ratio of the Auger surface and fluorescence detector energies plotted versus the aerosol transmission to the shower maximum $X_{\max }$. A fit to a straight line (solid red line) is consistent with the slope of zero. A negative slope would correspond to underestimated aerosol effects and a positive slope would indicate overestimated aerosol effects.

\subsection{SD and FD energy comparison}

Since the Auger and TA fluorescence detectors (FDs) provide a reliable calorimetric reconstruction of the UHECR energy, one can assess the nonlinearities of the Auger and TA surface detector (SD) energies by comparing the SD and FD energy reconstructions of the same cosmic-ray events.

Figure 7 shows the ratios of the TA SD energy to the TA FD energy plotted versus the energy for the events that have been simultaneously seen by the TA FD and the TA SD. The comparison has been done for two methods of reconstruction of the TA SD energy: using the attenuation correction derived from proton Monte Carlo showers simulated with QGSJET-II.3 (left) and using the modelindependent, constant intensity cut, attenuation correction [10] (right). As can be seen from Figure 7, the size of a possible nonlinearity of the TA energy is constrained to $-2 \% \pm 9 \%$. In [10], it is also shown that the TA SD constant intensity cut method and the shower attenuation correction derived from the TA SD Monte Carlo yield energies that agree at a $\sim 3 \%$ level with no net bias.

The Pierre Auger Collaboration has conducted this study also. Due to its large exposure, the calibration parameters used to convert the shower size at ground level into energy are determined with a statistical uncertainty at the $1 \%$ level and it is also possible to study the linearity of the energy in different declination bands. The high precision attained in the energy determination is shown in Figure 8, where the ratio of the Auger SD to FD energy is plotted versus the energy for different declination ranges. As can be seen from Figure 8, the deviations from unity are less than $2 \%$ per decade of energy. In addition, Figure 9 shows that such nonlinearities are less than $1 \%$ when the Auger SD to FD ratio above $10^{19} \mathrm{eV}$ is plotted versus declination. 

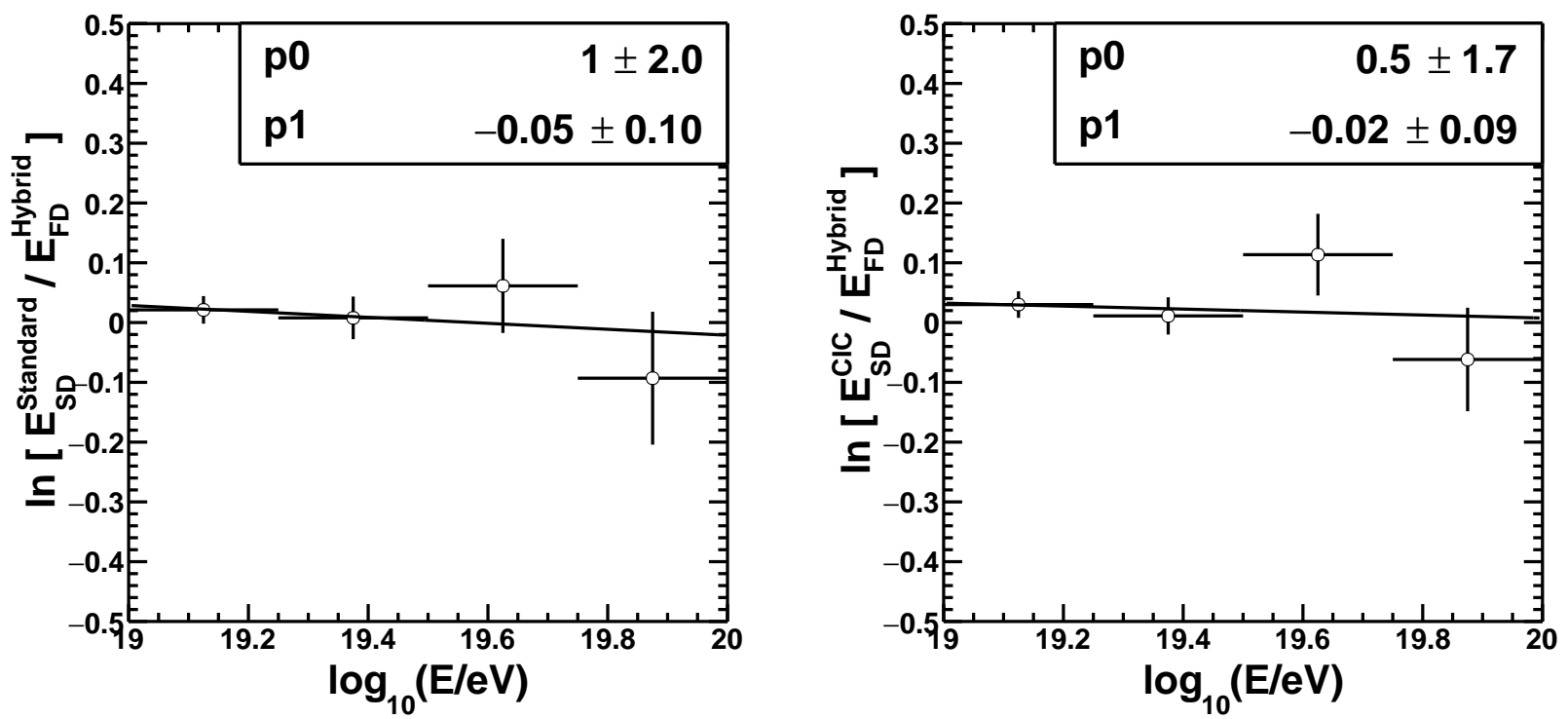

Figure 7. Natural logarithm of the energy reconstructed by the TA SD and the energy reconstructed by the TA FD for the (hybrid) events that have been seen both by the TA FD and SD in common, plotted versus the logarithm of energy. The left panel shows the SD and FD energy comparison for the standard, Monte Carlo-based reconstruction of the TA SD energy, and the right panel shows the same for the constant intensity cut (CIC) reconstruction of the TA SD energy. Fits to the straight lines yield slopes that are well within their fitting uncertainties.
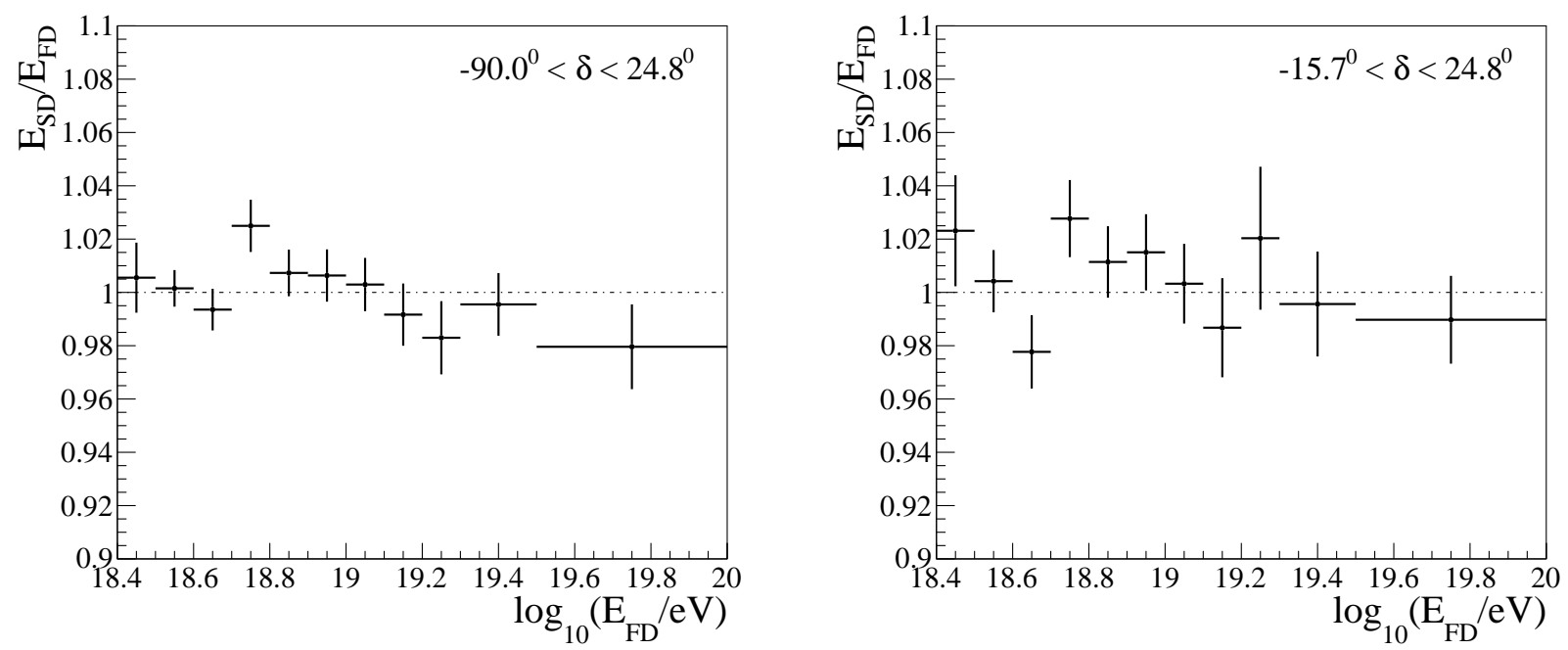

Figure 8. Ratio of the energies reconstructed by the Auger SD and FD plotted versus the logarithm of energy. The left panel shows the Auger SD and FD energy ratio for the entire Auger field of view, and the right panel shows the ratio of SD to FD energies for the Auger-TA common declination band.

\subsection{Alternative SD energy reconstruction methods}

To further assess the systematic effects of our reconstructions on our spectrum results, both Auger and TA have recalculated their surface detector spectra using alternative methods. In the past, TA has been calculating the SD energy spectrum using the attenuation correction derived from the CORSIKA QGSJET-II.3 proton Monte Carlo, while the Pierre Auger Collaboration has been using the attenuation correction obtained with the constant intensity cut method applied to only one energy threshold. We now show our spectrum results calculated applying the constant intensity cut method to the TA SD and an energydependent constant intensity cut method for the Auger SD.

Figure 10 shows the comparison of the TA SD spectra, in the two declination bands of interest, that have been calculated using the Monte Carlo-based attenuation correction and the constant intensity cut method [10]. The TA SD spectra calculated by the two different methods are in excellent agreement, as expected from the $3 \%$ - level 


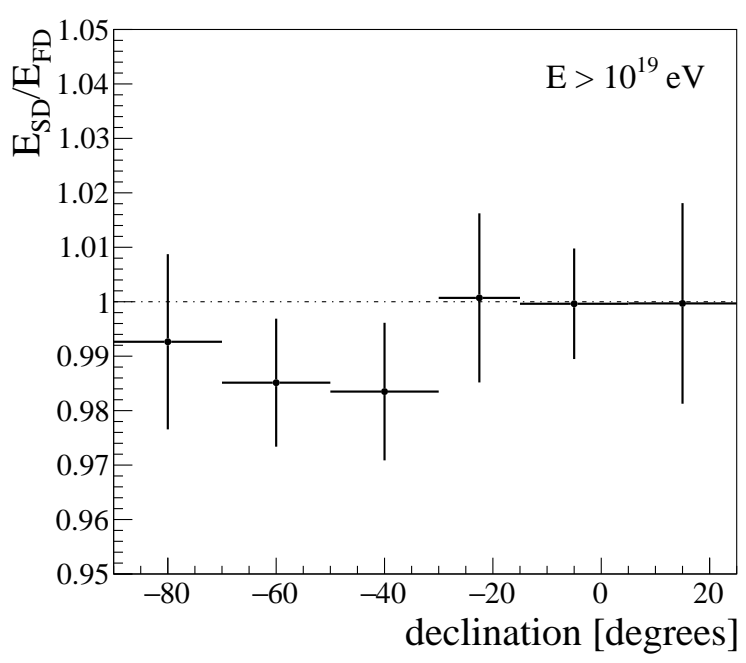

Figure 9. Ratio of the energies reconstructed by the Auger SD and FD plotted versus the event declination.

agreement of the TA SD energies calculated by the two methods [10].

The energy spectrum was checked by the Pierre Auger Collaboration by devising an attenuation correction based on a constant intensity cut method that depends on the energy. In the left panel of Figure 11, constant intensity cut (CIC) curves for the Auger data are shown for three energy thresholds. In the right-hand panel the small differences between the spectra introduced by using the energydependent $\mathrm{CIC}$ are shown. The maximum possible nonlinearity, arising from the use of the classic, single energy threshold CIC method, is of the order of $2 \%$.

Tables 1 and 2 show the summary of the energydependent systematic uncertainties of the Auger and TA energies. As can be seen from the Tables 1 and 2, the net energy dependent biases of TA and Auger are constrained to $-0.3 \pm 9 \%$ and $\pm 3 \%$, respectively.

Table 1. Sources of energy-dependent reconstruction bias in TA

\begin{tabular}{ll}
\hline Source of nonlinearity & $\begin{array}{l}\text { Amount } \\
\text { (\% per decade) }\end{array}$ \\
\hline FD Invisible energy & $1 \% \pm 1 \%$ \\
FD Fluorescence yield & $-1 \% \pm 1 \%$ \\
FD Aerosols & $1.7 \% \pm 1 \%$ \\
SD and FD comparison & $-2 \% \pm 9 \%$ \\
Net & $-0.3 \% \pm 9 \%$ \\
\hline
\end{tabular}

\section{Summary}

We have compared the TA and Auger surface detector spectra at the highest energies in the common declination band. We have found that there is a relative nonlinearity between the TA and Auger energies of the order of $20 \%$ per decade of energy above $10 \mathrm{EeV}$. If the Auger and $\mathrm{TA}$ energies are rescaled by the constant factors $+5.2 \%$
Table 2. Sources of energy-dependent reconstruction bias in Auger

\begin{tabular}{ll}
\hline Source of nonlinearity & $\begin{array}{l}\text { Amount } \\
\text { (\% per decade) }\end{array}$ \\
\hline Aerosols & $\pm 1 \%$ \\
Calibration & $\pm 1 \%$ \\
SD and FD comparison & $\pm 2 \%$ \\
Constant Intensity Cut & $\pm 2 \%$ \\
Net & $\pm 3 \%$ \\
\hline
\end{tabular}

and $-5.2 \%$ and adjusted, respectively, by additional $+10 \%$ per decade and $-10 \%$ per decade nonlinearity corrections above $10 \mathrm{EeV}$, the TA and Auger spectra come to an agreement. We have investigated the systematic uncertainties of TA and Auger that would produce the energy-dependent biases in their energy spectra, and we have found that such biases are constrained to $-0.3 \pm 9 \%$ for TA and $\pm 3 \%$ for Auger. 

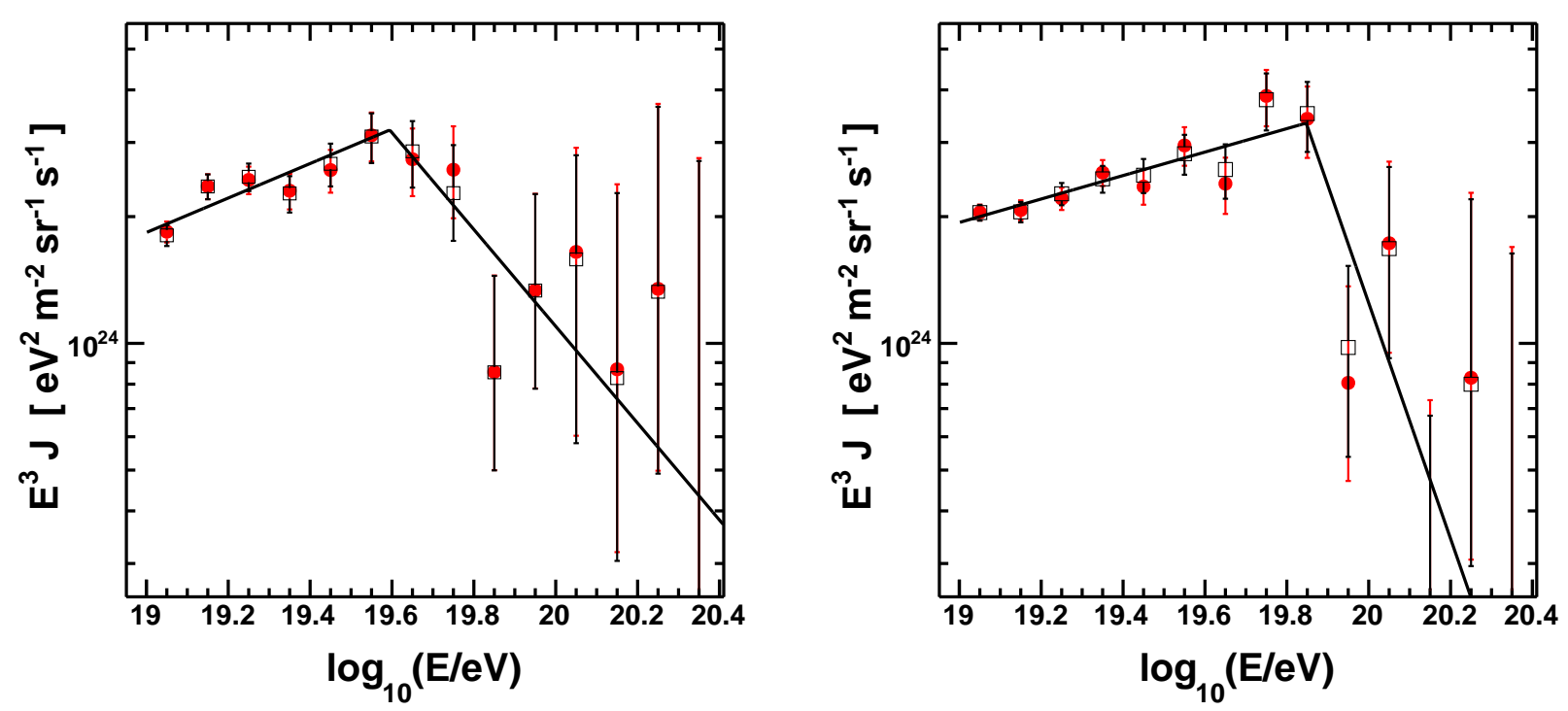

Figure 10. TA energy spectra calculated by the constant intensity cut method (red filled circles) and the traditional, Monte Carlo-based shower attenuation correction (open squares). The left panel is the comparison made in the Auger-TA common declination band, $-15.7^{\circ}<\delta<24.8^{\circ}$, and the right panel shows the comparison for the higher declination band, $24.8^{\circ}<\delta<90^{\circ}$.
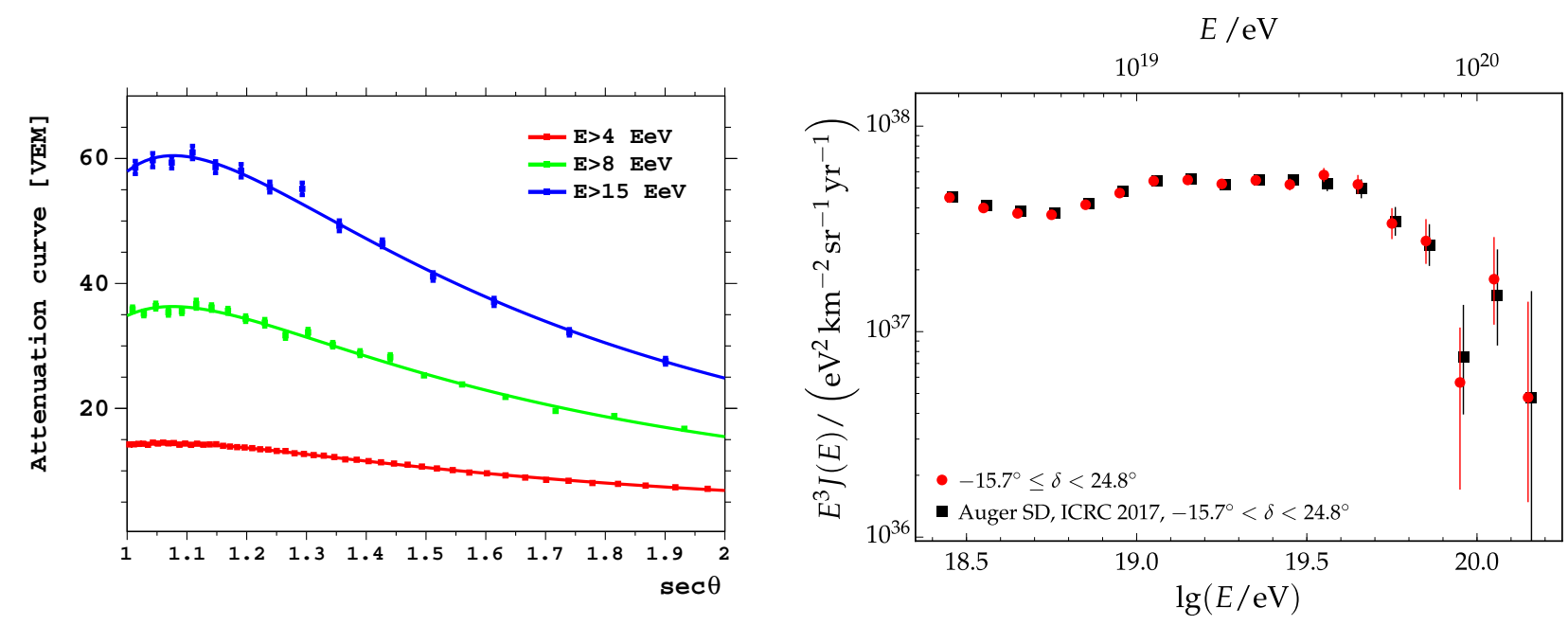

Figure 11. Left: Attenuation curves using the constant intensity cut method for the Auger SD made for multiple energy thresholds. Right: Comparison of the Auger SD spectra calculated using the standard Auger method (black squares) and the new, energy-dependent constant intensity cut method (red circles). 


\section{References}

[1] A. Aab et al., (Pierre Auger Collaboration), Nucl. Instrum. Meth. A 798 (2015) 172.

[2] T. Abu-Zayyad et al., (Telescope Array Collaboration), Nucl. Instrum. Meth. A 689 (2013) 87.

[3] T. Abu-Zayyad et al., (Telescope Array Collaboration), Astropart. Phys. 109 (2012) 39.

[4] H. Tokuno et al., (Telescope Array Collaboration), Nucl. Instrum. Meth. A 676 (2012) 54.

[5] F. Fenu, for the Pierre Auger Collaboration, Proc. 35th ICRC 2017, Bexco, Busan, Korea, PoS(ICRC2017)486.

[6] Y. Tsunesada, et. al., for the Telescope Array Collaboration, Proc. 35th ICRC 2017, Bexco, Busan, Korea, PoS(ICRC2017)535.

[7] B. R. Dawson et al., for the Telescope Array and Pierre Auger Collaborations, Proc. 2012 Int. Conf. Ultra-High Energy Cosmic Rays (UHECR2012), EPJ
Web of Conferences 53 (2013) 01005.

[8] T. AbuZayyad et al., for the Telescope Array and Pierre Auger Collaborations, Proc. 2016 Int. Conf. Ultra-High Energy Cosmic Rays (UHECR2016), JPS Conf. Proc. 19, 011003 (2018)

[9] D. Ivanov et al., for the Telescope-Array and Pierre Auger Collaborations, Proc. 35th ICRC 2017, Bexco, Busan, Korea, PoS(ICRC2017)498.

[10] D. Ivanov, for the Telescope Array Collaboration, UHECR 2018 Proceedings.

[11] V. Verzi, for the Pierre Auger Collaboration, in Proc. 33rd ICRC 2013, Rio de Janeiro, Brazil, arXiv:1307.5059 [astro-ph.HE].

[12] W. Hanlon, for the Telescope Array Collaboration, UHECR 2018 Proceedings.

[13] M. Malacari, for the Pierre Auger Collaboration, Proc. 35th ICRC 2017, Bexco, Busan, Korea, PoS(ICRC2017)398. 